\title{
Enhancement of parametric pumping due to Andreev reflection
}

\author{
Jian Wang, Yadong Wei, Baigeng Wang, and Hong Guo ${ }^{\text {a) }}$ \\ Department of Physics, The University of Hong Kong, Pokfulam Road, Hong Kong, China
}

(Received 4 June 2001; accepted for publication 21 September 2001)

\begin{abstract}
We report properties of parametric electron pumping in the presence of a superconducting lead. Due to a constructive interference between the direct reflection and the multiple Andreev reflection, the pumped current is greatly enhanced. For both quantum point contacts and double barrier structures at resonance, we obtain exact solutions in the weak pumping regime showing that $I_{p}^{\mathrm{NS}}=4 I_{p}^{N}$, which should be compared with the result of conductance $G_{\mathrm{NS}}=2 G_{N}$. Numerical results are also provided for the strong pumping regime showing interesting Andreev assisted pumping behavior. (C) 2001 American Institute of Physics. [DOI: 10.1063/1.1421236]
\end{abstract}

Current can flow under zero bias when two system parameters of a nanostructure are varied in a cyclic fashion. The physics of this parametric electron pump have been analyzed by several authors. ${ }^{1,2}$ Recently, it has been realized experimentally by Switkes et al. ${ }^{3}$ confirming many of the theoretical predictions. So far, investigations on parametric pumping are focused on normal nanostructures. It will be interesting to study a hybrid structure where a superconducting lead is present. In the presence of normal conductorsuperconductor (NS) interface, an incoming electron-like excitation can be Andreev reflected as a hole-like excitation. This current doubling effect gives rise to the relation for conductance $G_{\mathrm{NS}}=2 G_{N}$ for hybrid quantum point contacts and quantum dots at resonance. ${ }^{4}$ For dirty NS contacts, de Jong and Beenakker ${ }^{5}$ found that the shot noise at subgap voltages is also doubled with respect to its value in normal state which has been confirmed experimentally. ${ }^{6}$ To further explore how Andreev reflection modifies quantum interference in the normal state, we have investigated the parametric pumping phenomenon in the presence of a superconducting lead. We find that due to the quantum interference of the direct reflection and the multiple Andreev reflection, the pumped current is greatly enhanced. For quantum point contact and quantum dot at resonance, we obtained a relation for the pumped current, $I_{p}^{\mathrm{NS}}=4 I_{p}^{N}$ in the weak pumping regime. Numerical results are presented in the strong pumping regime showing interesting Andreev assisted pumping behavior which can be verified experimentally.

We consider a parametric pump which consists of a double barrier tunneling structure ${ }^{7}$ attached to a normal lefthand side lead and a superconducting right-hand side lead. The double barrier structure is modeled by potential $V(x)$ $=V_{1} \delta(x+a)+V_{2} \delta(x-a)$, where $V_{1}=V_{0}+V_{p} \sin (\omega t)$ and $V_{2}=V_{0}+V_{p} \sin (\omega t+\phi)$, and $V_{p}$ is the pumping amplitude. We further apply a gate voltage $v_{g}$ to control the energy level of the structure. The units are fixed by setting $\hbar=2 m=1$ in the following analysis. ${ }^{8}$ At low frequencies, the adiabatic pumped current in the presence of superconducting leads is ${ }^{1,9,10}$

\footnotetext{
${ }^{a)}$ Also at: Center for the Physics of Materials and Department of Physics, McGill University, Montreal, PQ, Canada H3A ZT8.
}

$$
I_{p}^{\mathrm{NS}}=\frac{\omega q}{\pi} \int_{0}^{\tau} d \tau\left[\frac{d N_{L}}{d V_{1}} \frac{d V_{1}}{d t}+\frac{d N_{L}}{d V_{2}} \frac{d V_{2}}{d t}\right]
$$

where the quantity $d N_{L} / d V$ is the injectivity ${ }^{11,12}$ given, at zero temperature, by

$$
\frac{d N_{L}}{d V_{j}}=\frac{1}{2 \pi} \operatorname{Im}\left[\mathcal{S}_{e e}^{*} \partial_{V_{j}} \mathcal{S}_{e e}-\mathcal{S}_{h e}^{*} \partial_{V_{j}} \mathcal{S}_{h e}\right],
$$

where the first term is the injectivity of the electron due to the external parameter, ${ }^{11,12}$ i.e., the partial density of states (DOS) for an electron coming from left-hand side lead and exiting the system as an electron, and the second term is the injectivity of a hole, i.e., the DOS for a hole coming from left-hand side lead and exiting the system as an electron.

For the hybrid nanostructure, the scattering matrix $\mathcal{S}_{e e}$ and $\mathcal{S}_{h e}$ are given by ${ }^{4,13}$

$$
\hat{\mathcal{S}}=\hat{S}_{11}+\hat{S}_{12}\left(1-\hat{R}_{I} \hat{S}_{22}\right)^{-1} \hat{R}_{I} \hat{S}_{21},
$$

where $\hat{\mathcal{S}}$ is a $2 \times 2$ scattering matrix for NS structure with matrix element $\mathcal{S}_{\mu \nu}(\mu, \nu=e, h)$ and $\hat{S}_{11}(E)=(I / 2)\left[S_{11}(E)\right.$ $\left.+S_{11}^{*}(-E)\right]+\left(\sigma_{z} / 2\right)\left[S_{11}(E)-S_{11}^{*}(-E)\right]$ where $S_{11}$ is the usual scattering matrix for double barrier structure. $\hat{R}_{I}$ $=\alpha \sigma_{x}$ is the $2 \times 2$ scattering matrix at the NS interface with off diagonal matrix element $\alpha$. Here $\alpha=(E$ $\left.-i \nu \sqrt{\Delta^{2}-E^{2}}\right) / \Delta$ with $\nu=1$ when $E>-\Delta$ and $\nu=-1$ when $E<-\Delta$. In Eq. (3), the energy $E$ is measured relative to the chemical potential $\mu$ of the superconducting lead. Equation (3) has clear physical meaning. ${ }^{13}$ The first term is the direct reflection from the normal scattering structure and the second term can be expanded as $\hat{S}_{12} \hat{R}_{I} \hat{S}_{21}$ $+\hat{S}_{12} \hat{R}_{I} \hat{S}_{22} \hat{R}_{I} \hat{S}_{21}+\ldots$ which is clearly the multiple Andreev reflection in the hybrid structure. From Eq. (3) we obtain the well known expressions for the scattering matrix $\mathcal{S}_{e e}$ and $\mathcal{S}_{h e}{ }^{4}$

$$
\mathcal{S}_{e e}(E)=S_{11}(E)+\alpha^{2} S_{12}(E) S_{22}^{*}(-E) M_{e} S_{21}(E)
$$

and

$$
\mathcal{S}_{h e}(E)=\alpha S_{12}^{*}(-E) M_{e} S_{21}(E)
$$

with $M_{e}=\left[1-\alpha^{2} S_{22}(E) S_{22}^{*}(-E)\right]^{-1}$. In the following, we first present the exact result for the pumped current in the 
weak pumping regime and then study the general situation numerically. In the weak pumping regime, Eq. (1) can be expanded to the lowest order in $V_{p}$,

$$
I_{p}^{\mathrm{NS}}=\frac{\omega q \sin \phi V_{p}^{2}}{\pi} \operatorname{Im}\left[\partial_{V_{1}} \mathcal{S}_{e e}^{*} \partial_{V_{2}} \mathcal{S}_{e e}-\partial_{V_{1}} \mathcal{S}_{h e}^{*} \partial_{V_{2}} \mathcal{S}_{h e}\right]
$$

as compared with the expression for the normal structure, ${ }^{1,10}$

$$
I_{p}^{N}=\frac{\omega q \sin \phi V_{p}^{2}}{\pi} \operatorname{Im}\left[\partial_{V_{1}} S_{11}^{*} \partial_{V_{2}} S_{11}+\partial_{V_{1}} S_{21}^{*} \partial_{V_{2}} S_{21}\right],
$$

where we set $V_{p}=0$ in $\mathcal{S}_{\nu \mu}$ and $S_{i j}$ after the partial derivatives. We further assume that the Fermi energy is close to the chemical potential of superconducting lead, so $E \sim 0$ and $\alpha$ $\sim-i$. Under this condition, $\mathcal{S}_{h e}$ and hence $\partial_{V_{j}} \mathcal{S}_{h e}$ are pure imaginary numbers for general $V_{j}(t)$. As a result, $\mathcal{S}_{h e}$ does not contribute to $I_{p}^{\mathrm{NS}}$ in Eq. (1) as long as $E=0 .{ }^{14}$ The only contribution comes from $\mathcal{S}_{e e}$ which is the superposition of the direct reflection and the multiple Andreev reflection. We will consider two cases: (a) a quantum point contact, e.g., $V_{0}=0$; and (b) the double barrier quantum dot at resonance. For both cases, $S_{11}=0$ in the absence of pumping voltage. Therefore, from Eq. (4) we have

$$
\partial_{V_{1 / 2}} \mathcal{S}_{e e}=\partial_{V_{1 / 2}} S_{11}-S_{12}^{2} \partial_{V_{2 / 1}} S_{11}^{*},
$$

where we have used the fact that $\partial_{V_{1}} S_{22}=\partial_{V_{2}} S_{11}$. Using Fisher-Lee relation ${ }^{15} S_{\alpha \beta}=-\delta_{\alpha \beta}+i \sqrt{v_{\alpha} v_{\beta}} G_{\alpha \beta}^{r}$ and the Dyson equation $\partial_{V_{j}} G_{\alpha \beta}^{r}=G_{\alpha j}^{r} G_{j \beta}^{r},{ }^{16}$ we have $\partial_{V_{1}} S_{11}$ $=i v G_{11}^{r} G_{11}^{r}=-i /(2 k) \quad$ and $\quad \partial_{V_{2}} S_{11}=i v G_{12}^{r} G_{21}^{r}=-i /$ (2k) $S_{12}^{2}$ with the velocity $v=2 k$. So for both cases (a) and (b) we have constructive interference between direct reflection and the multiple Andreev reflection $\partial_{V_{j}} \mathcal{S}_{e e}=2 \partial_{V_{j}} S_{11}$ which gives a pumped current $I_{p}^{\mathrm{NS}}=4 I_{p}^{N}$ with $I_{p}^{N}=$ $\pm \omega q V_{p}^{2} \sin (4 k a) /\left(4 \pi k^{2}\right)$, where the plus sign is for quantum point contact since $S_{12}=e^{2 i k a}$, and the minus sign corresponds to resonant tunneling since $S_{12}=e^{-2 i k a}$.

In the general situation, the pumped current can be calculated numerically using Eq. (1). ${ }^{17}$ Since the pumped current is proportional to $\omega$, we set $\omega=1$ for convenience. In the left-hand side inset of Fig. 1, we plot the ratio $I_{p}^{\mathrm{NS}} / I_{p}^{N}$ as a function of pumping strength $V_{p} / V_{0}$ for $V_{0}=79.2$ at the resonant point. We see that as the pumping amplitude increases, the constructive interference effect is suppressed. At small pumping strength, the ratio is about four which agrees with our theoretical analysis. At larger pumping strength, this ratio decreases to the value below two. Similar behavior is seen for the quantum point contact. Figure 1 shows the pumped current as a function of Fermi energy $E_{F}$ for different pumping amplitudes. For $V_{0}=79.2$, we have chosen $v_{g}$ $=-9.39$ so that one resonant level in the quantum dot is aligned with the chemical potential $\mu_{s}$ of the superconducting lead in the absence of pumping voltage $V_{p}$. For comparison, we also plot the Andreev reflection coefficient when $V_{p}=0$. Several observations are in order: (1) the pumped current is peaked near the resonant level showing clearly a resonant behavior. This is because the pumped current [Eq. (1)] is proportional to the DOS of the system which reaches its maximum near the resonance. (2) As the pumping amplitude $V_{p}$ increases, the pumped current increases. (3). The pumped current has two asymmetric peaks. To understand

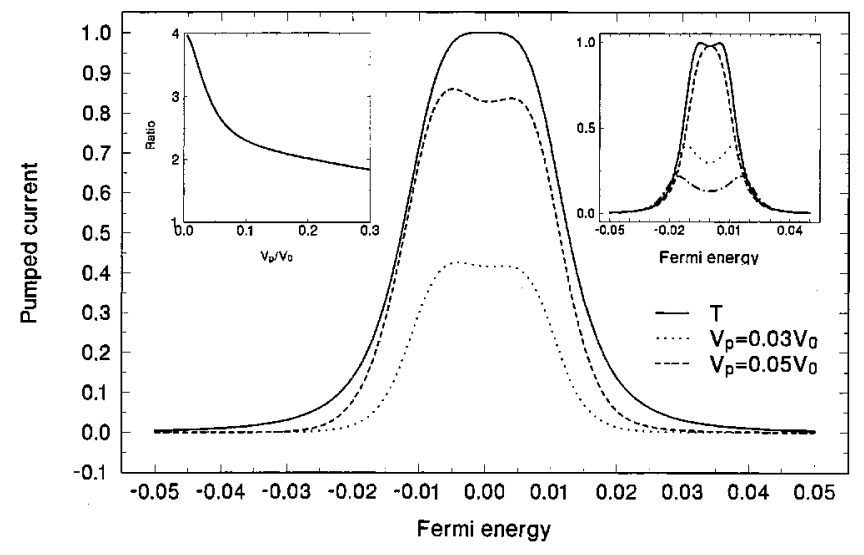

FIG. 1. The pumped current versus $E_{F}$ at different pumping amplitudes: $V_{p}=0.03 V_{0}, 0.05 V_{0}$. The left-hand side inset: $I_{p}^{N S} / I_{p}^{N}$ versus $V_{p} / V_{0}$ at resonant point. The right-hand side inset: Andreev reflection coefficient $T_{A}$ as a function of $E_{F}$ with $V_{p}=0.05 V_{0}$ at different pumping time: $t=\pi / 4$ (dotteddashed line), $t=\pi / 2$ (dotted line), $t=3 \pi / 4$ (solid line), and $t=7 \pi / 4$ (dashed line). System parameters in Fig. 1 and the insets: $V_{0}=79.2, v_{g}=-9.39, \phi$ $=\pi / 2$, and $\Delta=20$.

this, we plot in the right-hand side inset of Fig. 1 the Andreev reflection coefficient versus $E_{F}$ at different moments in one pumping cycle. From this inset, we observe that the Andreev reflection coefficient gives one or two peaks depending on the configuration of the system. This behavior can be understood from the Breit-Wigner form of the resonant Andreev reflection $T_{A}$ through a single level $E_{0}=0$ (measured relative to $\mu_{s}$ ): ${ }^{18,19}$

$$
T_{A}=\frac{\Gamma_{L}^{2} \Gamma_{R}^{2}}{4\left(E^{2}+\Gamma \Delta_{\Gamma} / 4\right)^{2}+\Gamma_{L}^{2} \Gamma_{R}^{2}},
$$

where $\Delta_{\Gamma}=\Gamma_{L}-\Gamma_{R}$ and $\Gamma=\Gamma_{L}+\Gamma_{R}$. We see that $T_{A}$ shows two peaks when $\Gamma_{L}<\Gamma_{R}$ and just one peak otherwise. Note that in one pumping cycle half of the configurations corresponds to $\Gamma_{L}<\Gamma_{R}$, therefore two pumped current peaks show up in Fig. 1 because from Eq. (1) the pumped current is obtained through the integral over all the configurations in one pumping cycle. Finally, the reason that two peaks are asymmetric is mainly due to the energy dependence of the self energy. If $\mu_{s}$ is right in the middle of two resonant levels ( $E_{1}$ and $\left.E_{2}\right)$, i.e., $\mu=\left(E_{1}+E_{2}\right) / 2$, then the electron coming from normal lead with incident energy $E_{1}$ tunnels into the structure through the resonant level $E_{1}$ and Andreev reflected as a hole back to the quantum dot through the resonant level $E_{2}$ with a Copper pair created in the superconducting lead. We now examine the Andreev assisted pumping through two levels. For $V_{0}=79.2$ and $v_{g}=-23.48$, there are two resonant levels inside the subgap $\left(\mu_{s}=0\right)$ at $E_{1}=14.09$ and $E_{2}$ $=-14.09$. Hence, strong Andreev reflections can occur near $E_{F}=E_{1}$. Figure 2 shows the two level pumped current versus Fermi energy (solid line). For comparison, we also plot the corresponding Andreev reflection coefficient versus Fermi energy when the $V_{p}$ is switched off (see inset of Fig. 2). Similar to Fig. 1, the pumped current also shows strong resonant behavior with smaller amplitude (compare Fig. 1 dotted-dashed line). We found two peaks of pumped current around $E_{1}$, one is near the resonant energy and the other one is shifted to a smaller energy with larger current. Although it is similar to Fig. 1 but has a different origin. This is due to AIP license or copyright, see http://apl.aip.org/api/copyright.jsp 


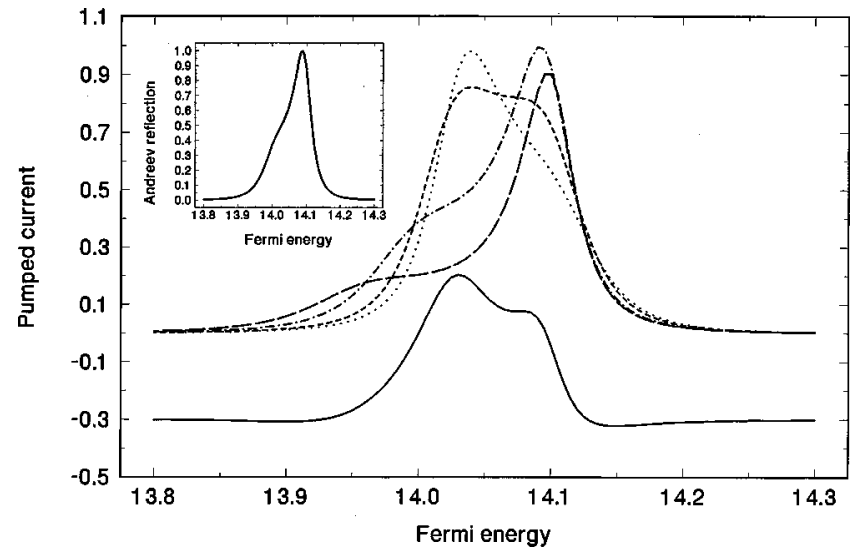

FIG. 2. The two level pumped current $I_{p}$ as a function of Fermi energy (solid line) with $V_{p}=0.05 V_{0}$ and $v_{g}=-23.481$. We also plot $T_{A}$ at several pumping time: $t=\pi / 4$ (dotted line), $t=\pi / 2$ (dashed line), $t=3 \pi / 4$ (dotteddashed line), and $t=\pi$ (long dashed line). The pumped current has been offset by 0.3 for illustrating purpose. Inset: $T_{A}$ versus Fermi energy when $V_{p}=0$. Other parameters are the same as Fig. 1.

the fact that when the pumping gate is turned on, the barrier heights and hence the resonant level change with time. To confirm this, we also plot the Andreev reflection coefficients $T_{A}$ at several instants of one pumping cycle in the same figure. The peaks of $T_{A}$ shift around the energy level at a different pumping time and give the behavior of pumped current. We have also calculated the pumped current for other system parameters and confirmed that the behavior of pumped current shown here is generic.

In summary, in the presence of the superconducting lead, the pumped current is greatly enhanced due to the quantum interference of direct reflection and multiple Andreev reflection. In the weak pumping regime, we have the relation $I_{p}^{\mathrm{NS}}$ $=4 I_{p}^{N}$ for both quantum point contact and the resonant tunneling structure. Interesting Andreev assisted pumping behaviors are revealed as well.
The authors gratefully acknowledge support by a RGC grant from the SAR Government of Hong Kong under Grant No. HKU 7215/99P. One of the authors (H.G.) is supported by NSERC of Canada and FCAR of Québec.

${ }^{1}$ P. W. Brouwer, Phys. Rev. B 58, R10135 (1998).

${ }^{2}$ F. Zhou, B. Spivak, and B. L. Altshuler, Phys. Rev. Lett. 82, 608 (1999); T. A. Shutenko, I. L. Aleiner, and B. L. Altshuler, Phys. Rev. B 61, 10366 (2000).

${ }^{3}$ M. Switkes, C. Marcus, K. Capman, and A. C. Gossard, Science 283, 1905 (1999).

${ }^{4}$ C. W. J. Beenakker, Rev. Mod. Phys. 69, 731 (1997).

${ }^{5}$ M. J. M. de Jong and C. W. J. Beenakker, Phys. Rev. B 49, 16070 (1994).

${ }^{6}$ X. Jehl, P. Payet-Burin, C. Baraduc, R. Calemczuk, and M. Sanquer, Phys. Rev. Lett. 83, 1660 (1999); X. Jehl, M. Sanquer, R. Calemczuk, and D. Mailly, Nature (London) 405, 50 (2000).

${ }^{7}$ Our analysis can also be applied the two-dimensional quantum dot similar to the experiment of Ref. 3, but with leads connected to the dot through narrow constrictions (play the role of barrier) controlled by a gate voltage.

${ }^{8}$ For the GaAs system with $a=1000 \mathrm{~A}$, the energy unit is $E=56 \mu \mathrm{eV}$.

${ }^{9}$ This formula can be derived using the Keldysh nonequilibrium Green's function method and we have assumed that the Coulomb blockade effect can be neglected.

${ }^{10} \mathrm{~A}$ factor of two has been included for spin.

${ }^{11}$ T. Gramespacher and M. Buttiker, Phys. Rev. B 61, 8125 (2000).

${ }^{12}$ J. Wang, Y. D. Wei, H. Guo, Q. F. Sun, and T. H. Lin, Phys. Rev. B 64, 104508 (2001).

${ }^{13}$ G. B. Lesovik, A. L. Fauchere, and G. Blatter, Phys. Rev. B 55, 3146 (1997).

${ }^{14}$ We assume a zero magnetic field. Since $S_{12}$ is symmetric with respect to $V_{1}$ and $V_{2}$, it does not contribute to $I_{p}^{N}$.

${ }^{15}$ D. S. Fisher and P. A. Lee, Phys. Rev. B 23, 6851 (1981).

${ }^{16}$ V. Gasparian, T. Christen, and M. Buttiker, Phys. Rev. A 54, 4022 (1996).

${ }^{17}$ M. K. Yip, J. Wang, and H. Guo, Z. Phys. B: Condens. Matter 104, 463 (1997).

${ }^{18}$ Y. D. Wei, J. Wang, H. Guo, H. Mehrez, and C. Roland, Phys. Rev. B 64, 115321 (2001).

${ }^{19}$ Y. D. Wei, J. Wang, and H. Guo, Phys. Rev. B 62, 9947 (2000). 